\title{
Child-Turcotte-Pugh and Model for End-stage Liver Disease Scores: Accuracy of Predictability for Mortality in Acute Variceal Hemorrhage
}

Kazim Abbas Virk ${ }^{1}$, Sana Tahir Virk ${ }^{2}$, Inayat Adil ${ }^{3}$, Shiza Tahir Virk ${ }^{4}$, Haseeb Noor ${ }^{5}$, Tehzeeb Zehra ${ }^{6}$

\begin{abstract}
Objective: To determine a score that best predicts the mortality of admitted patients within six weeks of Acute Variceal Hemorrhage (AVH).

Study Design: Cross sectional study.

Place and Duration of Study: The study was conducted at Inpatient Department of Gastroenterology Unit at Pakistan Institute of Medical Sciences (PIMS), Islamabad from 30 $0^{\text {th }}$ April 2017 to 30 $0^{\text {th }}$ October 2017.

Materials and Methods: The number of patients with AVH enrolled in this study were 223. A pretested questionnaire was used to gather the required information; Model for End-stage Liver Disease (MELD) and Child-Turcotte-Pugh (CTP) scores of each patient were recorded at the time of admission in the inpatient facility. The outcome was documented via a telephonic call at the end of six weeks. The primary outcome of the study was "mortality" at end of six weeks.

Results: The total number of participants were 223 , including $61 \%(n=136)$ males and $39 \%(n=87)$ females. The mean age was $52.4 \pm 13.96$. The overall mean value of CTP score was $9.6 \pm 2.8$ and mean value of MELD score was 19.3 \pm 6.7 , while the mean values of CTP and MELD among non-survivors were $12.9 \pm 2.1$ and 26.6 \pm 5.6 , respectively. After six weeks, the number of deaths were $27 \%(n=60)$. The MELD had positive predictive value (PPV) of $83.3 \%$, negative predictive value (NPV) of 96.8\%, sensitivity: $91.7 \%$, specificity: $93.3 \%$, diagnostic accuracy: $92.82 \%$, and positive likelihood ratio of 13.68. Similarly, CTP had PPV: $77.4 \%$, NPV: $92.5 \%$, sensitivity: $80 \%$, specificity: $91.4 \%$, diagnostic accuracy: $88.34 \%$ and positive likelihood ratio of 9.3 . The area under the curve (AUC)for MELD was 0.91, while CTP was 0.90.

Conclusion: The MELD score is better in its discriminative ability and more accurate in predicting six weeks mortality in patients with AVH than CTP score.
\end{abstract}

Key Words: Acute Variceal Hemorrhage, CTP, MELD, Mortality, NPV, PPV, Sensitivity, Specificity.

How to cite this: Virk KA, Virk ST, Adil I, Virk ST, Noor H, Zehra T. Child-Turcotte-Pugh and Model for End-stage Liver Disease Scores, Predictability for Mortality in Acute Variceal Hemorrhage in Terms of Accuracy. Life and Science. 2021; 2(1): 22-29. doi: http://doi.org/10.37185/LnS.1.1.133

This is an Open Access article distributed under the terms of the Creative Commons Attribution License (http://creativecommons.org/licenses/by/4.0), which permits unrestricted use, distribution, and reproduction in any medium, provided the original work is properly cited.

${ }^{1}$ Department of Medicine
Fazaia Medical College, Islamabad
${ }^{2}$ Department of Gastroenterology
HBS Medical College
HBS General Hospital, Islamabad
${ }^{3}$ Department of Gastroenterology
Rawal Institute of Medical Sciences, Rawalpindi
${ }^{4}$ Rawalpindi Medical University, Rawalpindi
${ }^{5}$ Department of Gastroenterology
Federal Government Polyclinic Hospital, Islamabad
${ }^{6}$ Department of Medicine
Shifa College of Medicine
Shifa International Hospital, Islamabad
C.....................................................................
Dr. Sana Tahir Virk
Department of Medicine
Fazaia Medical College, Islamabad
E-mail: stv082015@gmail.com

22
Funding Source: NIL; Conflict of Interest: NIL

Received: Jul 23, 2020; Revised: Nov 21, 2020

Accepted: Nov 25, 2020

\section{Introduction}

Liver cirrhosis is 14th most common cause of deaths in the world, ensuing about 1.03 million deaths annually. The mortality rate of liver cirrhosis fluctuates between $1-57 \%$ due to its various complications. ${ }^{1}$ Acute Variceal Hemorrhage (AVH) and portal hypertension are major complications, and comprises of about $70 \%$ of all upper gastrointestinal bleeding episodes in cirrhotic patients. ${ }^{2}$ Despite timely intervention, AVH carries about a six week mortality rate up to $23 \%{ }^{3}$ However, hospital mortality due to $\mathrm{AVH}$ was found $1.7 \%$ in one 
of national study. ${ }^{4}$

Cirrhosis comprises of two prognostic stagescompensated and decompensated. The decompensated cirrhosis is marked by development of complications like variceal hemorrhage, ascites, encephalopathy, jaundice, and hepatocellular carcinoma.

Different risk characteristics have been identified to develop scores that stratify patients in case of outcomes i.e., death and re-bleeding. The examples include Rockall, Child-Turcotte-Pugh (CTP), Model for End-stage Liver Disease (MELD) ${ }^{5}$, recalibrated MELD score, and Acute Physiology and Chronic Health Evaluation II (APACHE II).,

The most common, CTP and MELD scores are used in wide range of settings like intensive care, postsurgery, chronic Hepatitis B related cirrhosis, transjugular intrahepatic portosystemic shunt (TIPS), acute on chronic liver failure (ACLF) with $A V H$, and liver transplant candidates. The scores are compared for their prognostic accuracy with hospital base, 3month and 6-month mortality as the endpoints. ${ }^{1,7}$

In 1964, Child and Turcotte proposed score to estimate operative risk on patients of liver cirrhosis undergoing portosystemic shunt procedure for variceal hemorrhage. The score included variables like ascites, hepatic encephalopathy, nutritional status, total bilirubin, and serum albumin in the beginning. ${ }^{1}$ Later on, it was modified by Pugh and his colleagues by the addition of prothrombin time or international normalized ratio. ${ }^{8}$ Later, in 1973 it was termed as Child-Pugh classification after removal of nutritional status. ${ }^{9}$ The score was finally renamed as Child-Turcotte-Pugh (CTP) score and is use to predict the outcome of all cirrhotic patients regardless of surgery due to its ease and reliability, including livertransplantation and TIPS. ${ }^{7,10}$ The modified version of the score is given in the Table 1.

Several modifications were proposed in CTP score to improve its predictive value, for example-- inclusion of variceal status ${ }^{11}$, serum creatinine ${ }^{12}$, and recalibration to predict transplant free survival. ${ }^{13}$

The scores of CTP and MELD have been compared in different settings like variceal bleed, liver transplant, hepatocellular carcinoma, post shunt surgery and acute liver failure. Both have their limitations in terms of score variables and prognostic ability.

The Child-Pugh score comprises of subjective variables like ascites and hepatic encephalopathy which are subject to physicians' clinical judgment. The use of medications like diuretics and lactulose also alter actual disease prognosis by changing the clinical stage.

The third variable, international normalized ratio (INR) can be different according to the different reagents used in different laboratories.

\begin{tabular}{lccc}
\hline \multicolumn{3}{l}{ Table 1: Child-Turcotte-Pugh score } \\
\hline \multicolumn{4}{c}{ Numerical score } \\
Parameter & 1 & 2 & 3 \\
Ascites & None & Mild- & Moderate to \\
& & Moderate & Severe \\
Encephalopathy & None & $1-2$ & $3-4$ \\
Bilirubin & $<2.0$ & $2-3$ & $>3$ \\
Albumin & $>3.5$ & $2.8-3.5$ & $<2.8$ \\
Prothrombin & $1-3$ & $4-6$ & $>6.0$ \\
Time (Seconds & & & \\
increased) & & & \\
\hline
\end{tabular}

Class A :( 5-6); Class B: (7-9); Class C: (10-15)

The MELD score was initially generated to determine prognosis of trans jugular intrahepatic portosystemic shunts intervention patients in terms of survival. ${ }^{14,15}$ The current form of MELD score has three variables, total bilirubin, creatinine, and INR that are all objective and laboratory dependent. The MELD score has a weakness, as the INR does not provide sufficient information about coagulation disorder in the liver disease, and also it has inter-laboratory differences. ${ }^{16}$ The MELD score makes use of laboratory tests to add objective value to assess the disease severity as compared to empirical CTP score. Ascites and hepatic encephalopathy (HE) are not taken into account in MELD scores. ${ }^{17}$ The MELD score may underestimate the mortality risk of patients. ${ }^{14}$ hence, some researchers proposed that CTP score is feasible for daily clinical assessments of liver disease, and MELD score could be best in listing patients for liver transplant.

The designated formula for latest version of MELD score has the following variables.

MELD $=9.57 \times \log _{\mathrm{e}}[$ serum creatinine $(\mathrm{mg} / \mathrm{dL})]+$ $3.78 \times \log _{\mathrm{e}}[$ Total bilirubin $(\mathrm{mg} / \mathrm{dL})]+11.2 \times \log _{\mathrm{e}}[\mathrm{INR}]$ +6.43 (constant for liver Disease etiology)

Different studies have compared discriminative abilities of the MELD and CTP scores. The results seem to be still controversial. Few favored CTP score, while others MELD score. In systemic review and metanalysis MELD score is considered better to CTP 
score for prognosis in terms of survival, though MELD score is proved $63 \%$ better in predicting 3-month survival. ${ }^{14,17,18}$

The MELD score has several modifications which have been offered to improve the survival predictability in different liver related conditions. These modifications include HCC MELD for hepatocellular carcinoma ${ }^{19}$, MELD-Na (comprising of serum sodium of normal range ${ }^{20}$, Delta MELD for liver allocation ${ }^{21}$ integrated MELD incorporating serum sodium and age ${ }^{22}$, refit MELD (reallocating cut offs of serum creatinine and INR: 0.8-3.0 and 13 ,respectively) and MESO (ratio of MELD score to serum sodium). ${ }^{23}$

The CTP score has been used consistently to predict the risk of death due to acute variceal hemorrhage $(A V H)$. The MELD score is considered superior in assessment of liver disease severity rather than mortality from $\mathrm{AVH}^{24}$ The mortality for Index Bleed ranged from $15-80 \%$ for Child Class $B$ and C, $15 \%$ for child Class A. ${ }^{25}$

In another study, the prognostic abilities of MELD and CTP scores were assessed. The cut off in MELD score was $>23$ and Child score $>12$. Keeping mortality as reference standard, the sensitivity of MELD and CTP score was $100 \%$ and $83.3 \%$, respectively, whereas specificity was $81.3 \%$ and $92 \%$, respectively in the reference study. The PPV for MELD and CTP was $36.4 \%$ and $52.6 \%$, respectively. The NPV for MELD was $100 \%$ and $98.1 \%$ for CTP score, respectively. ${ }^{25}$

Pakistan is a country with widespread prevalence of cirrhosis. The access to tertiary care medical facilities with experienced endoscopists and standard equipment is limited. Although advanced endoscopic interventions are available at many centers, most government hospitals refer patients of AVH to teaching hospitals or private medical facilities because of either non-availability or malfunctioning of the equipment or owing to patient load. Based on the natural history of esophageal variceal hemorrhage $(E V H)$, the risk of re-bleeding has become similar to the non-bleeding esophageal varices in six weeks. ${ }^{2}$ There must be a scoring system available for triage, based on disease severity and prognosis, to designate such patients, at the time of admission in Emergency Department.

The aim of this study is to determine a score that best predicts the mortality of admitted patients within six weeks of $A V H$, so that, individualized management plan based on risk stratification by a score within appropriate time limits can be implied. It can be added to the emergency and inpatient management pathways. and assist in making a triage plan for patients, keeping in view the resources and disease burden.

\section{Materials and Methods}

Total 223 patients were enrolled at Gastroenterology Department of Pakistan Institute of Medical Sciences, Islamabad during $30^{\text {th }}$ April 2017 to $30^{\text {th }}$ October 2017, ranging of ages 18 to 80 years and of both genders. All the patients had decompensated chronic liver disease with index acute variceal hemorrhage and recurrent hemorrhage, diagnosed on upper gastrointestinal endoscopy. The sample size was calculated by WHO calculator and consecutive nonprobability sampling technique was employed in this cross-sectional validation study. The reference statistics for the calculation of sample size included confidence level: 95\%, expected sensitivity: $100 \%$ assuming $95 \%$ for the sample size, expected specificity: $81.3 \%$, expected prevalence: $23 \%$, desired precision: $5 \%$ for sensitivity and $10 \%$ for specificity. ${ }^{6}$

After a written consent from each patient information was collected through interviews on a pre-tested questionnaire by a trained data collector. The date and time of data entry was noted (Annex II). The endoscopy was performed to evaluate the presence of varices. The MELD and CTP scores were computed for each of the admitted case. The followup of patients was done after six weeks of initial presentation with $\mathrm{AVH}$. The primary outcome was taken as mortality within 6 weeks of initial presentation.

Data was evaluated by using IBM SPSS (Version: 24). The distribution of data was assessed by using Kolmogorov-Smirnov test. Descriptive statisticsfrequencies and percentages were estimated for the variables true positive (TP), true negative (TN), false positive (FP) false negative (FN), gender and primary outcome. The contingency tables were analyzed using $\chi^{2}$ test and significance level was kept of 0.05 . The Mann-Whitney $U$ test was used to compare the means, because the data was not normally distributed. A contingency table of $2 \times 2$ was 
constructed to calculate sensitivity, specificity, positive predictive value (PPV) and negative predictive value (NPV). The receiver operating characteristic (ROC) curve and likelihood ratio was measured to assess the discriminative ability. The values of area under the curve (AUC) $>0.8$ were considered clinically significant. Appropriate cut-off points were calculated from ROC. The effect modifiers were controlled by stratification and post stratification. Diagnostic Accuracy (DA) of the two scores was measured.

\section{Results}

The total participants enrolled in the study were 223 cases at different times for six months duration from $1^{\text {st }}$ May 2017 to $30^{\text {th }}$ Oct 2017. Out of these, 61\% $(n=136)$ were males and $39 \%(n=87)$ were females. The mean age was $52.4 \pm 13.95$ years ( $18-80$ years). The patients were designated by CTP scores at the time of enrollment. The obtained mean score was 9.6 \pm 2.8 SD. Similarly, patients were also assigned MELD scores at admission to ward. The mean value MELD score was 19.3 $\pm 6.7 S D$.

The patients were followed at six weeks via telephonic contacts and outcome was recorded for each patient. The $27 \%(n=60)$ were found dead and $73 \%(n=163)$ were those who survived. (figure 1 )

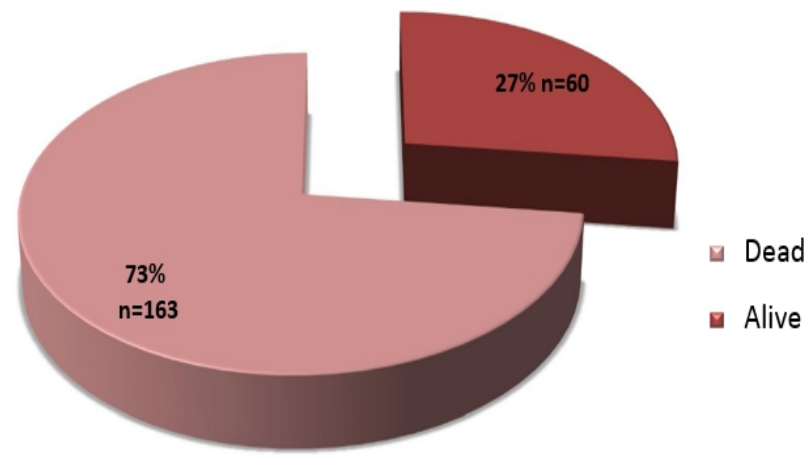

Fig 1: Outcome at 6 weeks

The Mann-Whitney $U$-test was employed to see if any difference exists between the mean of two scores for predicting the outcome. The test results were statistically significant with $p<0.01$ for both the score. There was also no statistically significant difference existed in observed and expected values in terms of outcome as evaluated by Chi square test $(p=0.20)$. The average values of MELD and CTP scores among survivors and non survivors are given in the Table 2.

\begin{tabular}{|c|c|c|}
\hline Scoring system & Survivors & Non-survivors \\
\hline MELD & $16.6 \pm 4.8$ & $26.6 \pm 5.6$ \\
\hline CTP & $8.4 \pm 2.0$ & $12.9 \pm 2.1$ \\
\hline
\end{tabular}

The percentages of TP, FP, FN, TN were calculated as $77.42 \%, 22.58 \%, 7.45 \%, 92.54 \%$, respectively. And for MELD score (Table.3), the percentages of TP, FP, FN, TN were calculated to be $83.33 \%, 16.6 \%, 3.18 \%$, and $96.81 \%$, respectively.

\begin{tabular}{|c|c|c|c|c|}
\hline & TP (\%) & $\mathrm{FP}(\%)$ & FN (\%) & TN (\%) \\
\hline MELD & 77.42 & 22.58 & 7.45 & 92.54 \\
\hline CTP & 83.33 & 16.6 & 3.18 & 96.81 \\
\hline
\end{tabular}

The Model's performance and discriminatory ability were assessed by sensitivity and specificity analysis (Table 4).

The discriminatory ability of MELD score is better than CTP score in predicting mortally at six weeks for Acute Variceal Hemorrhage. The kappa (measure of agreement) value for MELD is good $(k=0.82)$ but only fair for CTP score $(k=0.70)$.

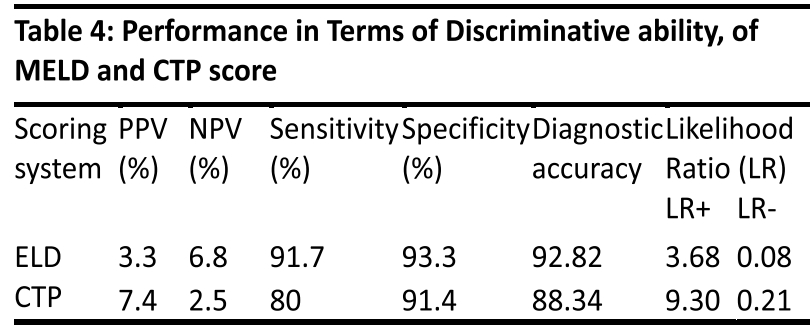

Patients were stratified with respect to age and gender, to determine discriminative ability of MELD and CTP scores (Table 5 and 6 ).

Table 5: The parameters for discriminative ability of MELD and CTP score for patients of age $>45$ years and $<45$ years

Age Scoring PV PV Sensitivity Specificity Diagnostic Likelihood (years) system (\%) (\%) (\%) (\%) accuracy Ratio (LR)

(\%) LR+ LR-

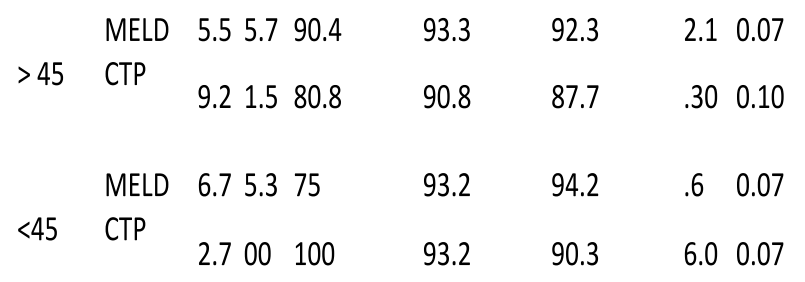


Table 6: The prognostic ability of two scores in Male and Female patients

\begin{tabular}{|c|c|c|c|c|c|}
\hline Gender & $\begin{array}{l}\text { Scoring } \\
\text { system }\end{array}$ & $\begin{array}{l}\text { PV PV Sensitivity } \\
\text { (\%) (\%) (\%) }\end{array}$ & $\begin{array}{l}\text { Specificity } \\
\text { (\%) }\end{array}$ & $\begin{array}{l}\text { Diagnostic } \\
\text { accuracy } \\
\text { (\%) }\end{array}$ & $\begin{array}{l}\text { Likelihood } \\
\text { Ratio (LR) } \\
\text { LR+ LR- }\end{array}$ \\
\hline \multirow{3}{*}{ Males } & MELD & 6.692 .9 & 91.5 & 91.9 & $\begin{array}{ll}0.9 & 0.08\end{array}$ \\
\hline & СТP & 7.58 .594 .4 & 92.8 & 93 & 3.10 .06 \\
\hline & MELD & 4.27 .188 .9 & 95.7 & 94.2 & $\begin{array}{ll}6.5 & 0.04\end{array}$ \\
\hline Females & CTP & 7.38 .594 .4 & 92.8 & 93 & 3.20 .07 \\
\hline
\end{tabular}

The AUC for MELD score was 0.91 that depicts the best discriminative accuracy (figure 2).

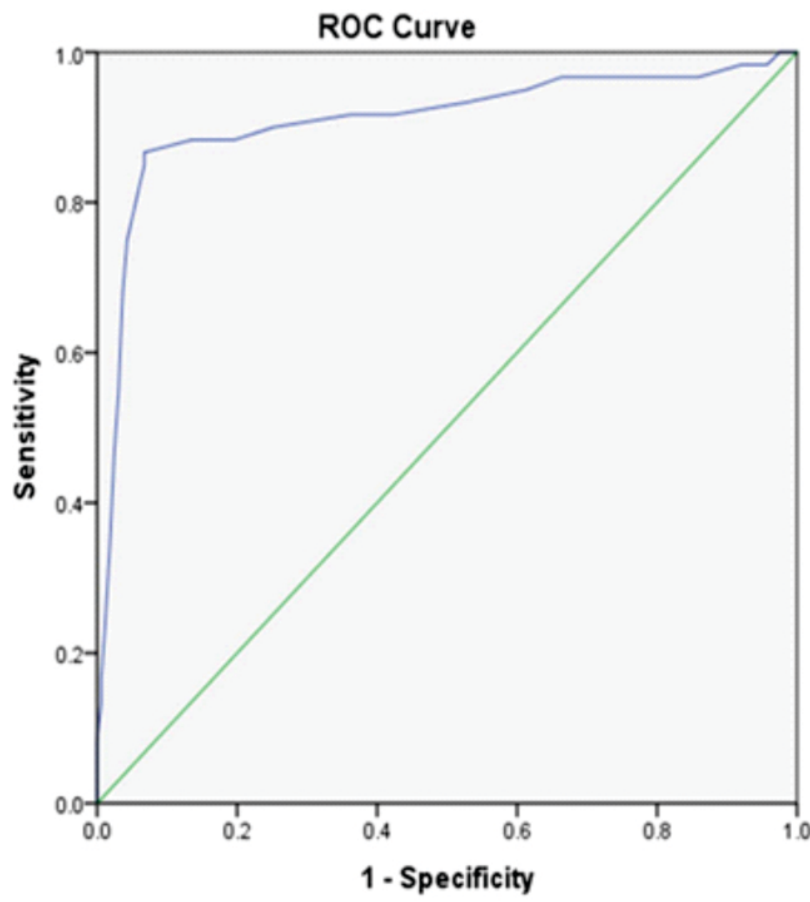

Diagonal segments are produced by ties.

True Positive Rate

False Positive Rate

Fig 2: Receiver Operating Curve for MELD Score

The AUC for CTP score was 0.90 , only slightly less than that for MELD. (figure 3 ).

The cut-off points were generated from the coordinates of the curve and appropriate cut-off points were selected using Youden Index. The cut-off for MELD score was found to be 23.5 with Youden index of 0.80 . The optimal cut-off point for CTP score was found to be 11.5 with Youden Index of 0.752 . The total mortality due to $\mathrm{AVH}$ for both the scores was found to be $26.9 \%$.

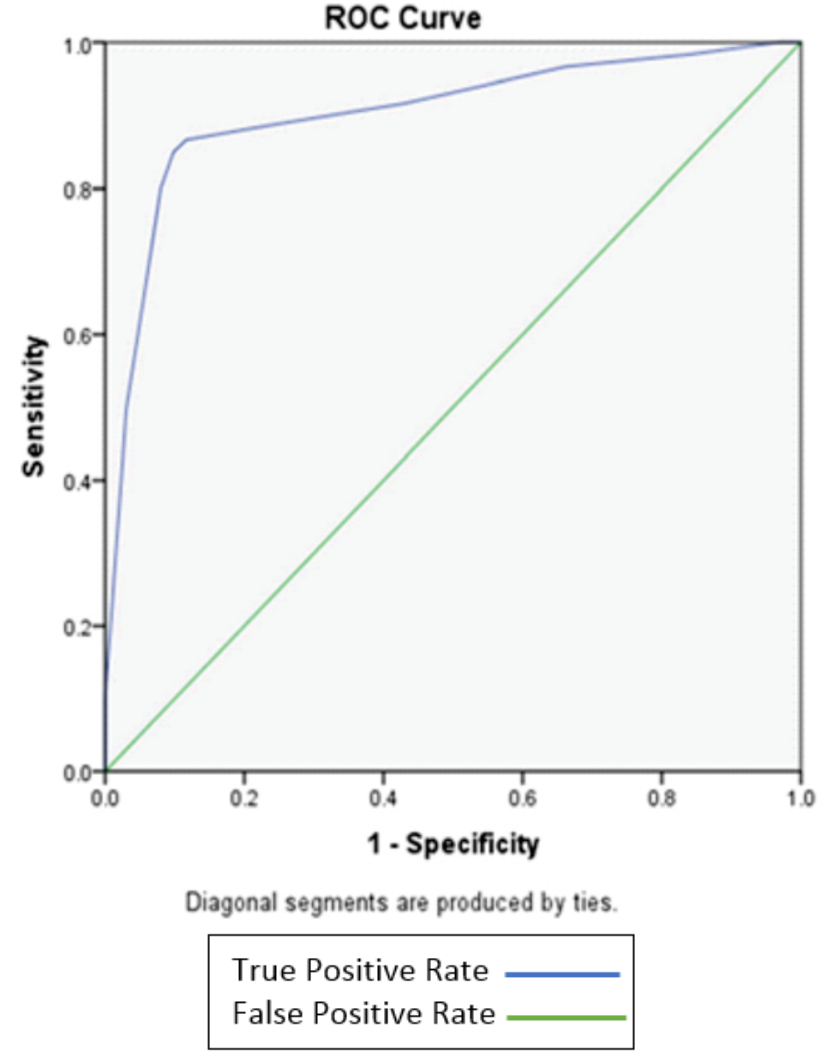

Fig 3: Receiver Operating Curve for CTP scores

\section{Discussion}

Selection of prognostically accurate score that can predict survival of patients of $\mathrm{AVH}$ at the time of admission as they are used to plan clinical management. It is extremely important to triage in resource limited settings where public hospitals are working out of their capacity owing to the exponential increase in the incidence of liver related diseases. It also limits the use of further interventions. Moreover, gastroenterologists can organize discussions with the families of the patients about the prognosis of the patients on evidencebased grounds.

The MELD and CTP scores are commonly employed scores among many that are used to assess the prognosis of liver disease and its complications. The MELD and CTP scores are compared for their discriminating ability in wide range of settings, but the results are debatable. ${ }^{1}$ Comparison of scores in patients of gastrointestinal hemorrhage and after TIPS are scrutinized in literature and the results have shown that in some studies MELD score is considered better while, in a few others, CTP score was found to be better. ${ }^{7}$ 
In this study, the primary outcome was six weeks mortality rate among cirrhotic patients with AVH of all severities. It was estimated $26.9 \%$, which was slightly higher as compared to the reference study where child class $C$ patients were enrolled and the mortality rate was $26 \%{ }^{3}$ Moreover, they excluded the patients of hepatocellular carcinoma and portal vein thrombosis that were not excluded in this study. This probably contributed to somewhat high mortality in the current study. In an earlier study the prevalence was $23 \%$, while in another study the mortality rate in re-bleeding cases was $38.3 \%$, that is significantly higher. ${ }^{2}$ This was probably because the patients with re-bleeding were at much advance stage in disease severity.

In current study, different parameters were selected to compare the scores like sensitivity, specificity, PPV, NPV, and likelihood ratios. ROC and AUC curves were depicted for both scores. The MELD score was established better regarding its prognostic and discriminative abilities as compared to CTP score. Various studies have been published to generate a best scoring system for prediction of mortality due to $\mathrm{AVH}$. In these studies, multiple scores were assessed in different clinical settings like re-bleeding cases in Intensive Care Units (ICU's), and for patients with different disease severities like hepatocellular carcinoma and portal vein thrombosis. These scores include those that predict liver disease severity in Outpatient Department and in critical patients.

In a study, MELD and CTP scores were compared with other scores used in critical care settings like Acute Physiologic Assessment and Chronic Evaluation II (APACHE II), Simplified Acute Physiology Score (SAPS II), and Sequential Organ Failure assessment (SOFA) scores. In this study, MELD score was found to be best with sensitivity of $100 \%$ and specificity of $81.3 \%$ The CTP score has sensitivity of $83.3 \%$, and specificity of $92 \%$. The PPV of MELD score was though only $36.4 \%$, which is much less than PPV of CTP score i.e., $52.6 \%$. The AUC for MELD was 0.89 and for CTP was 0.91. On contrary, the PPV of MELD score in our study was. $83.3 \%$ and of CTP score was $77.4 \%$. Conflicting to the above-mentioned study, the AUC for MELD in our study was 0.91 that is better than that of CTP i.e., 0.90 . These differences might be due to the study settings i.e., ICU and inclusion of other scores which perform much better in critical care settings. ${ }^{6}$
In another study, Albumin, International normalized ratio (INR), mental status, systolic blood pressure, Age $>65$ years (AIMS65) score, APACHE II, SOFA scores were compared along with MELD and CTP and concluded AIMS65 as best predictor of mortality and MELD performing better than CTP score. The sensitivity, specificity, PPV and AUC of MELD was $87.5 \%, 83.9 \%, 28 \%$ and 0.88 , respectively, while CTP had $87.5 \%, 73.2 \%, 18.9 \%$ and 0.82 respectively. ${ }^{9}$

Similarly, the two scores are extensively studied in cases of re-bleeding. Two recently published international and one local study have provided comparison of two scores in patients who were suffering from re- bleeding. One study only recorded MELD score in patients with rebleeding and hypovolemic shock and found that the value of MELD score $\geq 21.5$ is a better predictor of six weeks mortality after band ligation. The use of NonSelective Beta Blockers (NSBBs) is associated with lower six weeks mortality. The sensitivity, specificity, PPV, NPV of MELD calculated in this study were $78 \%$, $81 \%, 72 \%$ and $82 \%$, respectively. ${ }^{2}$

The value of MELD score $>18$, presence of hepatocellular carcinoma with portal vein thrombosis (PVT), active hemorrhage or white nipple sign visualization were best predictors of mortality up to six weeks after AVH in another study. ${ }^{8}$ The MELD, CTP, and Rockall scores were compared for estimating risk of re-bleeding and in hospital mortality and the AUC for predicting re-bleeding was calculated 0.419 for MELD, 0.52 for CTP, and 0.803 for Rockall. ${ }^{4}$

The CTP score was also established as a best stratifying risk factor for $\mathrm{AVH}$ in one of the studies. Both scores were found to be independent predictors of six weeks mortality. Although the AUC for MELD score (0.79) is greater than that for CTP $(0.75)$, but it was not significant $(p=0.27)$. The agreement between observed and predicted risk of six weeks mortality was best for CTP score $(p=0.45)$ and intermediate disagreement was reported for MELD score $(p=0.02)^{3}{ }^{3}$

This discussion would be incomplete without mentioning the results of a metanalysis published February 2016, which presented a systemic review of all the observational studies that compared MELD and CTP scores in AVH in terms of six weeks mortality. Of the 1095 studies, 119 were selected for this 
metanalysis. The 269 comparisons were done, of which 44 preferred MELD score, 16 were in favor of CTP score, 99 studies did not report any difference that was statistically significant. The Child-Pugh score was found to be more sensitive and less specific than MELD score in acute-on- chronic liver failure (ACLF) cases. The MELD score was found highly sensitive but had a smaller negative likelihood ratio than CTP score for critically ill cases but for surgery candidates CTP score is more specific than MELD score. ${ }^{1}$

\section{Conclusion}

In this study important confounding factors like type of management and interventions within six weeks, in-hospital mortality, rebleeding, severity of disease at time of admission, factors worsening natural history of disease like HCC and portal vein thrombosis are not considered. The need of intensive care unit is not mentioned as only inpatient cases were enrolled. Also, the measurement of Hepatic Venous Pressure Gradient (HVPG) could not be arranged due to limited resources.

Keeping in view these limitations, following are few recommendations that should be considered for future studies. In-hospital mortality of AVH and rebleeding are two other important factors which could be looked into with MELD and CTP scores. This was an observational study, further cohort and interventional studies are required to establish for the best scores for providing management guidelines. The treatment options and prophylaxis offered must be considered in future studies, so that a proper follow ups and early interventions can be planned. The need for ICU admission must be identified and other scores that are used in critical care settings must be considered and compared with MELD and CTP scores to further triage the patients on the basis of severity. The other medical and liver related conditions must be taken into account and all-causes of mortality should be the part of the studies, as these can be important contributing factors. The knowledge of the patients and history regarding variceal hemorrhage, treatment options available, natural history and prognosis with and without treatment must be further explored. Finally, proper diagnosis, monitoring of Hepatic Venous Pressure Gradient (HVPG)and its impact on six weeks mortality must be the part of future studies, for their cost effectiveness.

\section{REFERENCES}

1. Peng Y, Qi X, Guo X. Child-Pugh Versus MELD Score for the Assessment of Prognosis in Liver Cirrhosis: A Systematic Review and Meta-Analysis of Observational Studies. Medicine (Baltimore). 2016; 95: e2877.

2. Chen WT, Lin CY, Sheen IS, Huang CW, Lin TN, Lin CJ, et al. MELD score can predict early mortality in patients with rebleeding after band ligation for variceal bleeding. World J Gastroenterol. 2011;17:2120-5.

3. Fortune BE, Garcia-Tsao G, Ciarleglio M, Deng Y, Fallon MB, Sigal S, et al. Child-Turcotte-Pugh Class is Best at Stratifying Risk in Variceal Hemorrhage: Analysis of a US Multicenter Prospective Study. J Clin Gastroenterol. 2017; 51: 446-53.

4. Sarwar S, Khan AA, Tarique S. Comparison of MELD, Child Pugh score and Rockall score for predicting rebleeding and in-hospital mortality in patients of variceal bleeding. J Coll Physicians Surg Pak. 2008; 18: 524-5.

5. Vanderbrink BA, Cain MP, King S, Meldrum K, Kaefer M, Misseri $R$, et al. Is oral vitamin $B(12)$ therapy effective for vitamin B (12) deficiency in patients with prior ileocystoplasty? J Urol. 2010; 184 :1781- 5.

6. Freire $P$, Romaozinho JM, Amaro P, Ferreira M, Sofia C. Prognostic scores in cirrhotic patients admitted to a gastroenterology intensive care unit. Rev Esp Enferm Dig. 2011; 103: 177-83.

7. Zhou C, Hou C, Cheng D, Tang W, Lv W. Predictive accuracy comparison of MELD and Child-Turcotte-Pugh scores for survival in patients underwent TIPS placement: a systematic meta-analytic review. Int J Clin Exp Med. 2015; 8: 13464-72.

8. Pugh RN, Murray-Lyon IM, Dawson JL, Pietroni MC, Williams R. Transection of the oesophagus for bleeding oesophageal varices. Br J Surg. 1973; 60: 646-9.

9. Durand F, Valla D. Assessment of the prognosis of cirrhosis: Child-Pugh versus MELD. J Hepatol. 2005; 42: S100-7.

10. Fan $X$, Wen $M$, Shen $Y$, Wang $W$, Yang $X$, Yang L. Does adding variceal status to the Child-Turcotte-Pugh score improve its performance in predicting mortality in cirrhosis? Medicine (Baltimore). 2016; 95: e4884.

11. Giannini E, Botta F, Fumagalli A, Malfatti F, Testa E, Chiarbonello $B$, et al. Can inclusion of serum creatinine values improve the Child-Turcotte-Pugh score and challenge the prognostic yield of the model for end-stage liver disease score in the short-term prognostic assessment of cirrhotic patients? Liver Int. 2004; 24: 465-70.

12. Kaplan DE, Dai F, Skanderson M, Aytaman A, Baytarian M, D'Addeo K, et al. Recalibrating the Child-Turcotte-Pugh Score to Improve Prediction of Transplant-Free Survival in Patients with Cirrhosis. Dig Dis Sci. 2016; 61:3309-20.

13. World Gastroenterology Organisation Global Guidelines on esophageal varices. Source: Global Guidelines by WGO. $2014 \mathrm{Jan} ;[1-14 \mathrm{pp}$.]. Availablefrom: https://www.worldgastroenterology.org/guidelines/global -guidelines/esophageal-varices/esophageal-varicesenglish.

14. Malinchoc M, Kamath PS, Gordon FD, Peine CJ, Rank J, ter 
Borg PC. A model to predict poor survival in patients undergoing transjugular intrahepatic portosystemic shunts. Hepatology. 2000; 31: 864-71.

15. Kamath PS, Kim WR, Advanced Liver Disease Study G. The model for end-stage liver disease (MELD). Hepatology. 2007; 45: 797-805.

16. Trotter JF, Olson J, Lefkowitz J, Smith AD, Arjal R, Kenison J. Changes in international normalized ratio (INR) and model for endstage liver disease (MELD) based on selection of clinical laboratory. Am J Transplant. 2007; 7: 1624-8.

17. Cholongitas E, Papatheodoridis GV, Vangeli M, Terreni N, Patch D, Burroughs AK. Systematic review: The model for end-stage liver disease--should it replace Child-Pugh's classification for assessing prognosis in cirrhosis? Aliment Pharmacol Ther. 2005; 22: 1079-89.

18. Kim HJ, Lee HW. Important predictor of mortality in patients with end-stage liver disease. Clin Mol Hepatol. 2013; 19: 105-15.

19. Sala M, Varela M, Bruix J. Selection of candidates with HCC for transplantation in the MELD era. Liver Transpl. 2004; 10: S4-9.
20. Kim SY, Yim HJ, Lee J, Lee BJ, Kim DI, Jung SW, et al. [Comparison of CTP, MELD, and MELD-Na scores for predicting short term mortality in patients with liver cirrhosis]. Korean J Gastroenterol. 2007; 50: 92-100.

21. Freeman RB. Mathematical models and behavior: assessing delta MELD for liver allocation. Am J Transplant. 2004; 4: 1735-6.

22. Luca A, Angermayr B, Bertolini G, Koenig F, Vizzini G, Ploner $M$, et al. An integrated MELD model including serum sodium and age improves the prediction of early mortality in patients with cirrhosis. Liver Transpl. 2007; 13: 1174-80.

23. Magder LS, Regev A, Mindikoglu AL. Comparison of seven liver allocation models with respect to lives saved among patients on the liver transplant waiting list. Transpl Int. 2012; 25: 409-15.

24. Hunter SS, Hamdy S. Predictors of early re-bleeding and mortality after acute variceal haemorrhage. Arab J Gastroenterol. 2013; 14:63-7.

25. Mohammad AN, Morsy KH, Ali MA. Variceal bleeding in cirrhotic patients: What is the best prognostic score? Turk J Gastroenterol. 2016; 27: 464-9. 